\title{
Finite-Time Cooperative Tracking Control Algorithm for Multiple Surface Vessels
}

\author{
Jianfang Jiao and Mingyu Fu \\ College of Automation, Harbin Engineering University, Harbin, Heilongjiang 150001, China \\ Correspondence should be addressed to Mingyu Fu; fumingyu@hrbeu.edu.cn
}

Received 9 December 2013; Accepted 5 January 2014; Published 4 March 2014

Academic Editor: Shen Yin

Copyright ( 92014 J. Jiao and M. Fu. This is an open access article distributed under the Creative Commons Attribution License, which permits unrestricted use, distribution, and reproduction in any medium, provided the original work is properly cited.

\begin{abstract}
We investigate the problem of finite-time cooperative tracking for multiple surface vessels in the presence of external disturbances. A robust finite-time cooperative tracking algorithm based on terminal sliding-mode control is proposed for multiple surface vessels. In light of the leader-follower strategy, a virtual leader vessel is defined to provide reference point for other surface vessels to form the desired formation. Specifically, the proposed algorithm only requires the communication topology among the surface vessels to be a directed graph with a directed spanning tree. The robustness is achieved by compensating the upper bound of external disturbance in the control input, and the global finite-time stability is proved by Lyapunov stability theory. Finally, the effectiveness of the proposed finite-time cooperative tracking control algorithm is demonstrated by simulation results.
\end{abstract}

\section{Introduction}

With the rapid development of marine technology, the cooperative motion control for multiple vessels has received increasing attention during the last decades. The cooperative formation of multiple vessels has become popular for military and commercial applications. For example, coast patrol requires multiple vessels to perform cooperative tracking operation while maintaining a desired formation pattern. During winter, the tanker must be escorted by icebreakers, which requires the tanker to keep a fixed distance to the icebreakers. Besides, underway replenishment is performed by coordinating one or more supply vessels and the receiving vessel such that all vessels maintain the desired relative distances and hold the equal course and forward speed. These complicated operations of multiple vessels are carried out by moving collectively as a whole formation. Compared with individual vessel, cooperative operations of multiple vessels have higher operational efficiency, larger serve areas, better fault-tolerant property, and stronger robustness [1]. Based on these broad applications and several superiorities mentioned above, study on cooperative control algorithm for multiple surface vessels is important and significative.
With respect to the cooperative control issues, formation control as a special case, a large number of studies have been widely reported in existing publications. The formation strategies mainly include leader-follower strategy, virtual structures strategy, and behavioral strategy [2]. In order to achieve robustness and improve cooperative performance, some robust control approaches had been proposed, such as model predictive control [3], Lagrangian method [4], and null-space-based behavioral control [5]. Some advanced cooperative control approaches had also been investigated, such as graph theory [6], passivity-based control [7, 8], and hybrid control [9], to name just a few. In particular, the leader-follower strategy is utilized widely in practice due to its easy manipulation and implementation. For maritime applications, Kyrkjebø et al. proposed a leaderfollower synchronization algorithm to solve the ship underway replenishment, which realizes feedback control law by estimating velocity and acceleration of all ships based on nonlinear observers [10]. Breivik et al. proposed a guided leader-follower approach for ship formation control using integrator backstepping and cascade theory [11]. Thorvaldsen and Skjetne researched the formation control of fully actuated marine vessels and proposed group agreement protocols 
based on leader-follower strategy [7]. Overall, the cooperative task based on leader-follower strategy is achieved through that the appointed leaders track the predefined desired paths or trajectories, while the followers track the leaders. However, the main shortcoming of this formation strategy in the aforementioned studies is that it depends heavily on the leader. The formation task cannot be achieved if the leader has failure in the process of operations. To avoid this problem, the concept of virtual leader is introduced and used to solve the formation control of multiagent systems $[12,13]$. It is a good choice to design cooperative control algorithm based on the virtual leader strategy.

When multiple agents are to be coordinated to perform complicated task, information exchange between them is a necessary condition. In order to accomplish cooperative tracking operations, both position and velocity information need to be shared. In practice, the communication topology among these agents might be directed as a result of the external disturbances. That means one agent might receive the information from neighbors but cannot send his own information to the neighbors. Under directed communication topologies, Ren had studied the consensus tracking algorithm for multiagent with single-integrator kinematics [14]. Yu et al. provided a consensus algorithm for multiagent systems with nonlinear dynamics $[15,16]$. Zhang et al. studied the cooperative control problem of multiple uncertain Lagrangian systems [17]. Besides, Fu et al. proposed a coordinated formation control algorithm under directed communication topology for multiple surface vessels [18]. However, it is still a big problem to design a cooperative control algorithm under directed communication for the leaderfollower multiagent systems, especially in the case that the information of the leader is not available to all the followers; that is, only a portion of followers can communicate with the leader and the communication links are directed.

For marine control, finite-time control is quite desirable when considering the huge inertia of the surface vessels. Compared to asymptotic stability control, the convergence rate of finite-time control is faster, and the system with finitetime convergence has better disturbance rejection properties and robustness against uncertainties [19]. However, a common trait of the existing cooperative tracking control algorithms for multiple surface vessels is that they only provide asymptotic stability $[9,20]$. In other words, the cooperative operations can be achieved in infinite time, which may not be applicable to practical operation. So the finite-time cooperative control has received considerable attention. Wang and Xiao and Khoo et al. developed finite-time consensus algorithm for multiagent systems in [21, 22]. The finitetime formation control algorithms had been investigated for multiagent systems in $[23,24]$. Furthermore, both finitetime position consensus and collision avoidance problems had been investigated for multiple autonomous underwater vehicle [25]. The cooperative performance of multiple surface vessels is often influenced by the environmental disturbances; therefore, robust cooperative tracking algorithm is significative, and the real-time implementation of fault-tolerant control is also important [26-29]. The sliding-mode control is a better method for solving this problem, which possesses the robustness to external disturbances [30]. A robust tracking control algorithm is proposed based on sliding-mode control for a single surface vessel to achieve robustness to the wind, wave, and current environment disturbances in [31, 32]. The sliding-mode control approach is also used to design the robust cooperative control algorithm in $[20,33,34]$. The terminal sliding-mode control can be achieved by the finite-time cooperative operations [35], which motivates the research of the finite-time cooperative tracking for multiple surface vessels.

In this paper, the problem of robust cooperative tracking control for multiple surface vessels is considered, and the communication topology among these surface vessels is directed graph which has a directed spanning tree. The finitetime cooperative tracking control algorithm is designed using the terminal sliding-mode control method, and the desired formation configuration is achieved using the virtual leaderfollower strategy. The rest of this paper is organized as follows. In Section 2, the basic notations for the graph theory are introduced and the vessel mathematic model is established. Section 3 describes a detailed algorithm of the finite-time cooperative tracking control for multiple surface vessels. The simulation is carried out to demonstrate the validity of the proposed cooperative control algorithm in Section 4. At last, we draw conclusion in Section 5.

\section{Preliminaries}

2.1. Vessel Model. With respect to the surface vessels, only the motions on the surge, sway, and yaw are considered. If we define the generalized position and orientation which are expressed in the inertial reference frame as $\eta=[n, e, \psi]^{T}$, the linear-angular velocity vector expressed in the body-fixed reference frame is denoted as $v=[u, v, r]^{T}$. Then we can obtain the 3 degrees of freedom (DOF) mathematical model for the surface vessels as follows [36]:

$$
\begin{gathered}
\dot{\eta}=R(\psi) v, \\
M_{v} \dot{v}+C_{v}(v) v+D_{v}(v) v=\tau_{v}+R^{-T}(\psi) \omega,
\end{gathered}
$$

where $R(\psi)$ is a transformation matrix from the body-fixed reference to the inertial reference frame and the form is

$$
R(\psi)=\left[\begin{array}{ccc}
\cos (\psi) & -\sin (\psi) & 0 \\
\sin (\psi) & \cos (\psi) & 0 \\
0 & 0 & 1
\end{array}\right] .
$$

It is obvious that $R_{i}^{-1}\left(\psi_{i}\right)=R_{i}^{T}\left(\psi_{i}\right)$, for all $\psi_{i}$.

$M_{v}$ denotes a positive definite matrix of inertia mass which includes added mass. $C_{v}(v)$ is a matrix which arises from the coriolis and centripetal forces and $D_{v}(v)$ represents a damping matrix. The detailed expression of the above three matrices can be seen in [36]. $\tau_{v}$ represents the forces and torques input vector from the thruster system. $\omega$ denoted the forces and torques input vector from the external disturbances. And we assume that the disturbances are bounded; $|\omega|<\omega_{\max } \in \mathbb{R}^{3}$. 
In order to design the tracking controller for surface vessels in the sequel, the expression of vessel model can be transformed as

$$
M(\eta) \ddot{\eta}+C(\eta, \dot{\eta}) \dot{\eta}+D(\eta, \dot{\eta}) \dot{\eta}=\tau+\omega .
$$

The above expression is vessel mathematic model in the inertial reference frame, which is obtained by using the following transformations:

$$
\begin{gathered}
M(\eta)=R^{-T}(\psi) M_{v} R^{-1}(\psi), \\
C(\eta, \dot{\eta})=R^{-T}(\psi)\left[C_{v}(v)-M_{v} R^{-1}(\psi) \dot{R}(\psi)\right] R^{-1}(\psi), \\
D(\eta, \dot{\eta})=R^{-T}(\psi) D_{v}(v) R^{-1}(\psi), \\
\tau=R^{-T}(\psi) \tau_{v} .
\end{gathered}
$$

The vessel model as (3) holds the following properties.

(1) Inertia mass matrix $M(\eta)$ is symmetric positive definite and satisfies

$$
\lambda_{\min }(M) I \leq M(\eta) \leq \lambda_{\max }(M) I,
$$

where $\lambda_{\min }(M)$ represents the minimum eigenvalue of the matrix $M$ and $\lambda_{\max }(M)$ represents the maximal eigenvalue of the matrix $M$;

(2) $\dot{M}(\eta)-2 C(\eta, \dot{\eta})$ satisfies

$$
\eta^{T}(\dot{M}(\eta)-2 C(\eta, \dot{\eta})) \eta=0, \quad \forall \eta \in \mathbb{R}^{3}
$$

which means it is skew symmetric;

(3) $D(\eta, \dot{\eta})$ is positive definite matrix which satisfies

$$
\eta^{T} D(\eta, \dot{\eta}) \eta>0, \quad \forall \eta \neq 0 .
$$

2.2. Notations. In order to model the information transmit relationship between the group of surface vessels, several basic concepts of directed graph are given here [20]. If we define $v$ as a set of vertices and define $\varepsilon \in v^{2}$ as a set of edges, then we can represent a directed graph as $G=(\nu, \varepsilon)$. Furthermore, the edges of directed graph are directed. The directed edge $\langle i, j\rangle \in \varepsilon$ can represent the information that flows from vertex $j$ to vertex $i$, and $\langle j, i\rangle \in \varepsilon$ represents the information that flows from vertex $i$ to vertex $j$. Let $A \in \mathbb{R}^{n \times n}$ be the adjacent matrix of a directed graph $G$. The matrix $A$ is defined as follows: the off-diagonal entries are $a_{i j}=$ 1 if $\langle i, j\rangle \in \varepsilon$ and 0 ; otherwise, the diagonal entries are 0 . $D \in \mathbb{R}^{n \times n}$ is called the degree matrix, which is defined as follows: off-diagonal entries are 0 and diagonal entries are $d_{i i}=\sum_{j \neq i} a_{i j}$. The Laplacian matrix can be calculated as $L=D-A \in \mathbb{R}^{n \times n}$. The matrix $L=\left[l_{i j}\right] \in \mathbb{R}^{n \times n}$ is defined as follows: $l_{i i}=\sum_{j \neq i} a_{i j}, l_{i j}=-a_{i j}$.

Let one vertex represent one vessel in the group and the edges represent information exchange links by available directed communication; then the communication relationship between the group of vessels is described by a directed graph. Specially, in this paper we consider the communication topology as a directed graph with a directed spanning tree; that is, the digraph has at least one vertex with a directed path to all other vertexes.

Define the Kronecker product of two matrices $A \in \mathbb{R}^{n \times n}$ and $B \in \mathbb{R}^{p \times q}$ as

$$
A \otimes B=\left[\begin{array}{ccc}
a_{11} B & \cdots & a_{1 n} B \\
\vdots & \ddots & \vdots \\
a_{m 1} B & \cdots & a_{m n} B
\end{array}\right] \in \mathbb{R}^{m p \times n q} .
$$

The Kronecker product holds the following properties:

(1) $(A \otimes B)^{T}=A^{T} \otimes B^{T}$;

(2) $C(A \otimes B)=(C A) \otimes B=A \otimes(C B)$;

(3) $\left(A \otimes I_{p}\right)\left(C \otimes I_{p}\right)=A C \otimes I_{p}, I_{p} \in \mathbb{R}^{p \times p}$.

Given a variable vector $x=\left[x_{1}, \ldots, x_{n}\right]^{T} \in \mathbb{R}^{n}$ and an integer $\alpha$, define $x^{\alpha}=\left[x_{1}^{\alpha}, \ldots, x_{n}^{\alpha}\right]^{T}, \operatorname{diag}(x)=\left[\begin{array}{lll}x_{1} & & \\ & \ddots & \\ & & x_{n}\end{array}\right]$.

\subsection{Some Lemmas}

Lemma 1 (see [14]). Let the Laplacian matrix of a directed graph $G$ be defined as $L=\left[l_{i j}\right] \in \mathbb{R}^{p \times p}$, where $L$ is not necessarily symmetric. The Laplacian matrix satisfies the following conditions:

$$
l_{i j} \leq 0, i \neq j ; \quad \sum_{j=1}^{p} l_{i j}=0, \quad i=1, \ldots p .
$$

The Laplacian matrix $L$ of a directed graph $G$ has a simple zero eigenvalue with an associated eigenvector $1_{p}$, and all the other eigenvalues have positive real parts if and only if the directed graph has a directed spanning tree. Furthermore, if Laplacian matrix $L$ has a simple zero eigenvalue, then $\operatorname{Rank}(L)=p-1$.

Lemma 2 (see [37]). For the non-Lipschitz system

$$
\dot{x}=f(x), \quad f(0)=0, \quad x \in \mathbb{R}^{n},
$$

where $f(\cdot)$ is a continuous nonlinear function on an open neighborhood $U$ of the origin $x=0$ in $\mathbb{R}^{n}$. Suppose there exist a continuous function $V(x): U \rightarrow \mathbb{R}$, real numbers $C>0$ and $0<\alpha<1$, and an open neighborhood $U_{0} \subset U$ of $x=0$, such that

(1) $V(x)$ is positive definite;

(2) $\dot{V}(x)+C(V(x))^{\alpha} \leq 0, x \in U_{0} \backslash\{0\}$.

Then the origin $x=0$ is a finite-time stable equilibrium of system (10). Furthermore, if $U=U_{0}=\mathbb{R}^{n}$, the origin $x=0$ is a globally finite-time stable equilibrium of system (10). And the finite settling time satisfies $T \leq V^{1-\alpha}\left(x_{0}\right) / C(1-\alpha)$, where $x_{0}$ is the initial state of the system.

\section{Finite-Time Cooperative Tracking Controller Design}

In this section, we will design the finite-time cooperative tracking controller based on terminal sliding-mode control. 
Here we consider $n$ vessels to perform the cooperative tracking task with desired formation. And these vessels are identified by the index set $I=[1,2, \ldots n]$. We define the communication topology relationship among these vessels as a directed graph $G$; then the adjacent matrix of $G$ is

$$
A=\left[\begin{array}{cccc}
a_{11} & a_{12} & \cdots & a_{1 n} \\
\vdots & \vdots & \vdots & \vdots \\
a_{n 1} & a_{n 2} & \cdots & a_{n n}
\end{array}\right] \in \mathbb{R}^{n \times n} .
$$

The degree matrix is defined as $D=\operatorname{diag}\left\{\begin{array}{llll}d_{1} & d_{2} & \cdots & d_{n}\end{array}\right\} \in$ $\mathbb{R}^{n \times n}$; then we can know that the Laplacian matrix is $L=D$ A.

The desired formation pattern among the surface vessels is established based on the leader-follower strategy. The leader vessel is virtual and it is labeled by 0 . Then the communication topology among all the vessels (include the virtual leader) is described by a directed graph $\bar{G}$; the adjacent matrix of $\bar{G}$ is denoted as

$$
\bar{A}=\left[\begin{array}{cccc}
0 & 0 & 0 & 0 \\
a_{10} & a_{11} & \cdots & a_{1 n} \\
\vdots & \vdots & \vdots & \vdots \\
a_{n 0} & a_{n 1} & \cdots & a_{n n}
\end{array}\right] \in \mathbb{R}^{(n+1) \times(n+1)}
$$

The connected relationship between the leader vessel and the practical vessels is denoted by $B=\operatorname{diag}\left\{\begin{array}{llll}b_{1} & b_{2} & \cdots & b_{n}\end{array}\right\}$.

Remark 3. Consider the following.

$$
a_{i j}= \begin{cases}1, & \text { if }(j, i) \in \varepsilon \\ 0, & \text { otherwise }\end{cases}
$$

$$
b_{i}= \begin{cases}1, & \text { if vessel } i \text { can receive the leader's information } \\ 0, & \text { otherwise. }\end{cases}
$$

We assume that the position of the virtual leader vessel is denoted as $\eta_{0}$ and the desired trajectory of the whole formation is given by the leader vessel. Here we define the desired trajectory of the leader as $\eta_{d}$, where $\eta_{d}=\left[n_{d}(t), e_{d}(t), \psi_{d}(t)\right]^{T}$, $n_{d}(t), e_{d}(t)$ are sufficiently smooth functions, and the motion direction of the virtual leader vessel can be chosen as the tangential vector of its desired trajectory; that is, $\psi_{d}(t)=$ $\arctan \left(\dot{e}_{d}(t) / \dot{n}_{d}(t)\right)$. In order to form the desired formation, we define the relative distance between the $i$ th follower vessel and the virtual leader vessel as $l_{i}=\left[x_{0 i}, y_{0 i}, \psi_{0 i}\right]^{T}$ and $\psi_{0 i}=0$; then we can define the formation reference point of the $i$ th vessel as $x_{i}=\eta_{i}+l_{i}$. It is obvious that $l_{0}=0$; then $x_{0}=\eta_{0}$. In order to maintain the desired formation among these surface vessels, it is necessary for all the formation reference points to synchronize. That is, $x_{1}=\cdots=x_{i}=\cdots=x_{n}=\eta_{0}$. And the cooperative tracking while keeping the desired formation is achieved by $x_{1}=\cdots=x_{i}=\cdots=x_{n}=\eta_{d}$.

The virtual vessel is free to external disturbances, so the leader vessel model in the inertial reference frame can be written as

$$
M_{0}\left(\eta_{0}\right) \ddot{\eta}_{0}+C_{0}\left(\eta_{0}, \dot{\eta}_{0}\right) \dot{\eta}_{0}+D_{0}\left(\eta_{0}, \dot{\eta}_{0}\right) \dot{\eta}_{0}=\tau_{0} .
$$

Design the tracking control law $\tau_{0}$ using the backstepping control approach to make $\eta_{0} \rightarrow \eta_{d}$ as in the literature [20].

We assume that the position of the virtual vessel and its velocity are available to its neighbors only and the control force input $\tau_{0}$ is unknown to any practical vessels, but its upper bound $\bar{\tau}_{0}$ is available to its neighbors. The detailed design process of the finite-time cooperative tracking algorithm is as follows.

Define the relative position error of the formation reference point for the $i$ th vessel as

$$
\begin{aligned}
e_{1}^{i} & =\sum_{j=1}^{n} a_{i j}\left(x_{i}-x_{j}\right)+b_{i}\left(x_{i}-x_{0}\right) \\
& =\sum_{j=1}^{n} a_{i j}\left(\eta_{i}+l_{i}-\eta_{j}-l_{j}\right)+b_{i}\left(\eta_{i}+l_{i}-\eta_{0}\right) .
\end{aligned}
$$

Define the the relative velocity error of the formation reference point for for the $i$ th vessel in the inertial reference frame as

$$
\begin{aligned}
e_{2}^{i} & =\sum_{j=1}^{n} a_{i j}\left(\dot{x}_{i}-\dot{x}_{j}\right)+b_{i}\left(\dot{x}_{i}-\dot{x}_{0}\right) \\
& =\sum_{j=1}^{n} a_{i j}\left(\dot{\eta}_{i}-\dot{\eta}_{j}\right)+b_{i}\left(\dot{\eta}_{i}-\dot{\eta}_{0}\right) \\
& =\left(\sum_{j=1}^{n} a_{i j}\right) \dot{\eta}_{i}-\left(\sum_{j=1}^{n} a_{i j}\right) \dot{\eta}_{j}+b_{i} \dot{\eta}_{i}-b_{i} \dot{\eta}_{0} .
\end{aligned}
$$

Define the terminal sliding-mode surface of the $i$ th vessel as

$$
s_{i}=e_{1}^{i}+\left(e_{2}^{i}\right)^{\alpha}
$$

where $\left(e_{2}^{i}\right)^{\alpha}=\left[\left(e_{2}^{i}(1)\right)^{\alpha}\left(e_{2}^{i}(2)\right)^{\alpha}\left(e_{2}^{i}(3)\right)^{\alpha}\right]^{T}$. The realtime control input $\tau_{0}$ of the virtual leader is unknown to any following vessels due to time delay or information transmission failure in the communication channel; while the upper bound of the control input $\bar{\tau}_{0}$ is available to the adjacent vessels. The control input of each vessel can be chosen as

$$
\begin{aligned}
\tau_{i}=C_{i} & \left(\eta_{i}, \dot{\eta}_{i}\right) \dot{\eta}_{i}+D_{i}\left(\eta_{i}, \dot{\eta}_{i}\right) \dot{\eta}_{i} \\
+ & \sum_{j=1, j \neq i}^{n}\left(a_{i j}+b_{i}\right)^{-1} M_{i} \\
\times & \left\{\frac{\left(e_{2}^{i}\right)^{2-\alpha}}{\alpha}+\sum_{j=1, j \neq i}^{n} a_{i j} M_{j}^{-1}\right. \\
& \times\left[-C_{j}\left(\eta_{j}, \dot{\eta}_{j}\right) \dot{\eta}_{j}-D_{j}\left(\eta_{j}, \dot{\eta}_{j}\right) \dot{\eta}_{j}+\tau_{j}\right] \\
& +b_{i} M_{0}^{-1}\left[-C_{0}\left(\eta_{0}, \dot{\eta}_{0}\right) \dot{\eta}_{0}-D_{0}\left(\eta_{0}, \dot{\eta}_{0}\right) \dot{\eta}_{0}\right] \\
& -\operatorname{diag}\left(2 n M_{\min }^{-1} \omega_{\max }+b_{i} M_{\min }^{-1} \bar{\tau}_{0}+\kappa_{1}\right) \\
& \left.\times \operatorname{sign}\left(s_{i}\right)\right\},
\end{aligned}
$$


where $\kappa_{1} \in \mathbb{R}^{3 \times 1}$ is positive vector and $0<M_{\min } \leq$ $\min \left\{M_{1}, \ldots M_{n}\right\}$.

Theorem 4. Consider the vessel with the nonlinear model as in (1) and (3), if the communication topology among all the vessels (include the virtual leader) is a directed graph which has a directed spanning tree and the terminal sliding-mode surface is defined as (17), the control input force is chosen as (18). Then, the cooperative tracking of multiple surface vessels can be reached in finite time.

Proof. The Laplacian matrix of the communication graph among these surface vessels is

$$
L=D-A=\left[\begin{array}{ccc}
\left(\sum_{j=1}^{n} a_{1 j}\right)-a_{11} & \cdots & -a_{1 n} \\
\vdots & \ddots & \vdots \\
-a_{n 1} & \cdots & \left(\sum_{j=1}^{n} a_{n j}\right)-a_{n n}
\end{array}\right] .
$$

The connected relationship between the leader vessel and the practical vessels is denoted as

$$
B=\left[\begin{array}{lll}
b_{1} & & \\
& \ddots & \\
& & b_{n}
\end{array}\right] .
$$

If we define

$$
\begin{gathered}
E_{1}=\left[\begin{array}{lll}
e_{1}^{1} & \cdots & e_{1}^{n}
\end{array}\right]^{T} \in \mathbb{R}^{3 n \times 1}, \\
E_{2}=\left[\begin{array}{lll}
e_{2}^{1} & \cdots & e_{2}^{n}
\end{array}\right]^{T} \in \mathbb{R}^{3 n \times 1}, \\
\eta=\left[\begin{array}{lll}
\eta_{1} & \cdots & \eta_{n}
\end{array}\right]^{T} \in \mathbb{R}^{3 n \times 1}, \\
1_{n}=\left[\begin{array}{lll}
1, \ldots, & 1
\end{array}\right]^{T} \in \mathbb{R}^{n \times 1}, \\
I_{3}=\operatorname{diag}\left(\begin{array}{lll}
1 & 1 & 1
\end{array}\right) \in \mathbb{R}^{3 \times 3},
\end{gathered}
$$

then the error dynamics of multiple surface vessels can be written in terms of matrix and vector:

$$
\begin{gathered}
\dot{E}_{1}=E_{2}, \\
\dot{E}_{2}=\left[(L+B) \otimes I_{3}\right] \ddot{\eta}-\left(B \otimes I_{3}\right)\left(1_{n} \otimes \ddot{\eta}_{0}\right) .
\end{gathered}
$$

For representing conveniently, we define

$$
\begin{aligned}
& \eta=\left[\begin{array}{llll}
\eta_{1}^{T} & \eta_{2}^{T} & \cdots & \eta_{n}^{T}
\end{array}\right]^{T} ; \\
& l=\left[\begin{array}{llll}
l_{1}^{T} & l_{2}^{T} & \ldots & l_{n}^{T}
\end{array}\right]^{T} ; \\
& \tau=\left[\begin{array}{llll}
\tau_{1}^{T} & \tau_{2}^{T} & \cdots & \tau_{n}^{T}
\end{array}\right]^{T} ; \\
& M(\eta)=\operatorname{diag}\left(M_{1}\left(\eta_{1}\right) \cdots M_{n}\left(\eta_{n}\right)\right), \\
& C(\eta, \dot{\eta})=\operatorname{diag}\left(C_{1}\left(\eta_{1}, \dot{\eta}_{1}\right) \cdots C_{n}\left(\eta_{n}, \dot{\eta}_{n}\right)\right) \text {, } \\
& D(\eta, \dot{\eta})=\operatorname{diag}\left(D_{1}\left(\eta_{1}, \dot{\eta}_{1}\right) \cdots D_{n}\left(\eta_{n}, \dot{\eta}_{n}\right)\right) .
\end{aligned}
$$

We can redefine the error dynamics with the matrix or vector form with the vessel model; then we can obtain that

$$
\begin{aligned}
\dot{E}_{1}=E_{2}, & \\
\dot{E}_{2}= & {\left[(L+B) \otimes I_{3}\right] } \\
& \times\left[M^{-1}(\eta) \times(\tau+\omega-C(\eta, \dot{\eta}) \dot{\eta}-D(\eta, \dot{\eta}) \dot{\eta})\right] \\
- & \left(B \otimes I_{3}\right) \\
& \times\left\{1 _ { n } \otimes \left[M_{0}^{-1}\left(\eta_{0}\right)\right.\right. \\
& \left.\left.\times\left(\tau_{0}-C_{0}\left(\eta_{0}, \dot{\eta}_{0}\right) \dot{\eta}_{0}-D_{0}\left(\eta_{0}, \dot{\eta}_{0}\right) \dot{\eta}_{0}\right)\right]\right\} .
\end{aligned}
$$

The terminal sliding-mode variable vector can be written as $S=\left[\begin{array}{lll}s_{1} & \cdots & s_{n}\end{array}\right]^{T}$; then we can obtain that

$$
S=E_{1}+E_{2}^{\alpha}
$$

Consider the Lyapunov function

$$
V=\frac{1}{2} S^{T} S
$$

Differentiating $V$ with respect to time, we can obtain that

$$
\begin{aligned}
& \dot{V}=S^{T} \dot{S} \\
&=S^{T}\left[E_{2}+\right.\left.\alpha \operatorname{diag}\left(E_{2}^{\alpha-1}\right) \dot{E}_{2}\right] \\
&=S^{T}\left\{E_{2}\right.+\alpha \operatorname{diag}\left(E_{2}^{\alpha-1}\right) \\
& \times\left[\left((L+B) \otimes I_{3}\right)\right. \\
& \times\left(M^{-1}(\eta)(\tau+\omega-C(\eta, \dot{\eta}) \dot{\eta}-D(\eta, \dot{\eta}) \dot{\eta})\right) \\
&-\left(B \otimes I_{3}\right) \\
& \times\left(1 _ { n } \otimes \left(M_{0}^{-1}\left(\eta_{0}\right)\right.\right. \\
& \times\left(\tau_{0}-C_{0}\left(\eta_{0}, \dot{\eta}_{0}\right) \dot{\eta}_{0}\right. \\
&\left.\left.\left.\left.\left.\quad-D_{0}\left(\eta_{0}, \dot{\eta}_{0}\right) \dot{\eta}_{0}\right)\right)\right)\right]\right\} .
\end{aligned}
$$




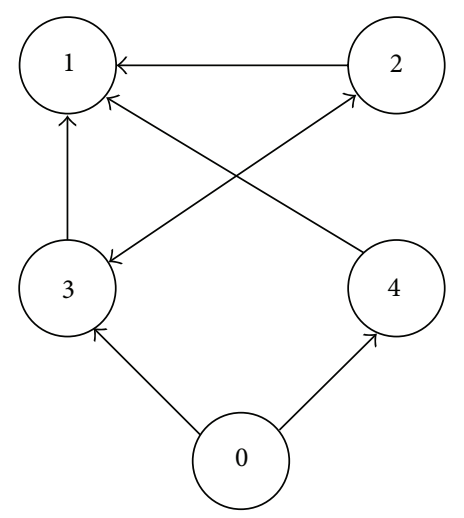

FIGURE 1: The information exchange topology among all the vessels.

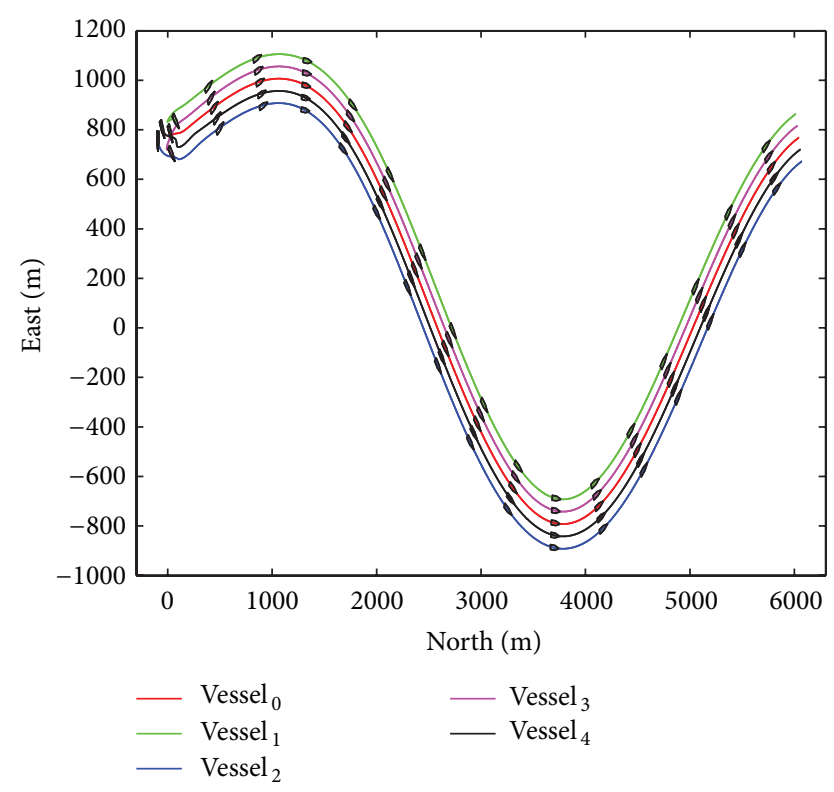

FIGURE 2: The dynamic trajectory of each vessel.

The control input vector of all these vessels can be written as

$$
\begin{aligned}
\tau= & C(\eta, \dot{\eta}) \dot{\eta}+D(\eta, \dot{\eta}) \dot{\eta}+\left[(D+B)^{-1} \otimes I_{3}\right] M \\
\times & \frac{\left(E_{2}\right)^{2-\alpha}}{\alpha}+\left(A \otimes I_{3}\right) M^{-1} \\
& \times(\tau-C(\eta, \dot{\eta}) \dot{\eta}-D(\eta, \dot{\eta}) \dot{\eta})
\end{aligned}
$$

$$
\begin{aligned}
& +\left(B \otimes I_{3}\right) \\
& \times\left(1_{n} \otimes M_{0}^{-1}\left(-C_{0}\left(\eta_{0}, \dot{\eta}_{0}\right) \dot{\eta}_{0}-D_{0}\left(\eta_{0}, \dot{\eta}_{0}\right) \dot{\eta}_{0}\right)\right) \\
& -\operatorname{diag}\left\{\operatorname{diag}\left(M_{\text {min }}^{-1}\left(2 n \omega_{\max }+b_{i} \bar{\tau}_{0}\right)+\kappa_{1}\right),\right. \\
& \left.\ldots, \operatorname{diag}\left(M_{\text {min }}^{-1}\left(2 n \omega_{\text {max }}+b_{i} \bar{\tau}_{0}\right)+\kappa_{1}\right)\right\} \\
& \quad \times \operatorname{sign}(S)\} .
\end{aligned}
$$

We can note that

$$
\begin{aligned}
I_{3 n}- & \left\{\left[(D+B)^{-1} \otimes I_{3}\right] M\left(A \otimes I_{3}\right) M^{-1}\right\} \\
= & M M^{-1}-\left\{\left[(D+B)^{-1} \otimes I_{3}\right] M\left(A \otimes I_{3}\right) M^{-1}\right\} \\
= & M\left[(D+B)^{-1} \otimes I_{3}\right]\left[(D+B) \otimes I_{3}\right] M^{-1} \\
& -\left\{\left[(D+B)^{-1} \otimes I_{3}\right] M\left(A \otimes I_{3}\right) M^{-1}\right\} \\
= & M\left[(D+B)^{-1} \otimes I_{3}\right]\left[(D+B) \otimes I_{3}-\left(A \otimes I_{3}\right)\right] M^{-1} \\
= & M\left[(D+B)^{-1} \otimes I_{3}\right]\left[(D+B-A) \otimes I_{3}\right] M^{-1} \\
= & M\left[(D+B)^{-1} \otimes I_{3}\right]\left[(L+B) \otimes I_{3}\right] M^{-1} .
\end{aligned}
$$

Then the control input can be rewritten as

$$
\begin{aligned}
\tau= & M\left((L+B)^{-1} \otimes I_{3}\right) \\
\times & \left((D+B) \otimes I_{3}\right) M^{-1} \\
\times & \{C(\eta, \dot{\eta}) \dot{\eta}+D(\eta, \dot{\eta}) \dot{\eta} \\
+ & \left((D+B)^{-1} \otimes I_{3}\right) M \\
& \times\left\{\frac{\left(E_{2}\right)^{2-\alpha}}{\alpha}+\left(A \otimes I_{3}\right) M^{-1}\right. \\
& \times(\tau-C(\eta, \dot{\eta}) \dot{\eta}-D(\eta, \dot{\eta}) \dot{\eta})+\left(B \otimes I_{3}\right) \\
& \times\left(1_{n} \otimes M_{0}^{-1}\left(-C_{0}\left(\eta_{0}, \dot{\eta}_{0}\right) \dot{\eta}_{0}-D_{0}\left(\eta_{0}, \dot{\eta}_{0}\right) \dot{\eta}_{0}\right)\right) \\
& \quad-\operatorname{diag}\left\{\operatorname{diag}\left(M_{\min }^{-1}\left(2 n \omega_{\max }+b_{i} \bar{\tau}_{0}\right)+\kappa_{1}\right),\right. \\
& \left.\quad \ldots, \operatorname{diag}\left(M_{\min }^{-1}\left(2 n \omega_{\max }+b_{i} \bar{\tau}_{0}\right)+\kappa_{1}\right)\right\} \\
& \quad \times \quad \operatorname{sign}(S)\}\} .
\end{aligned}
$$




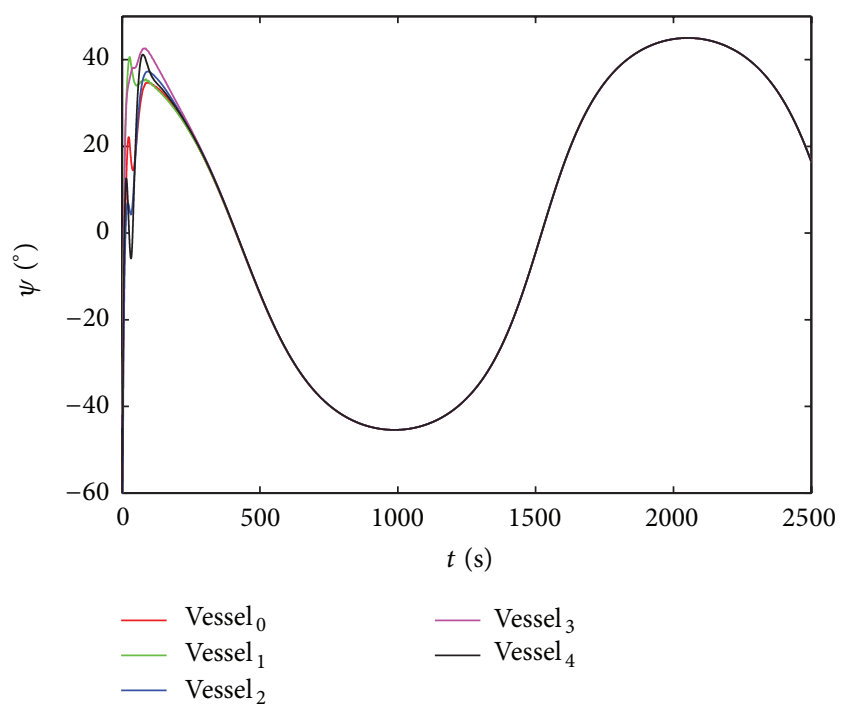

FIGURE 3: The heading consensus for these vessels.
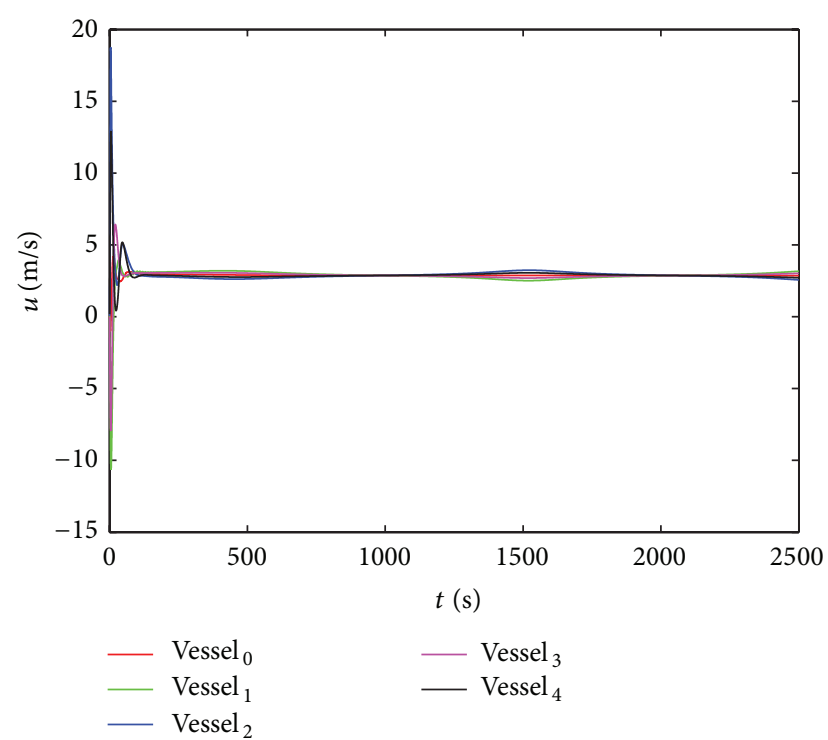

FIgURE 4: The surge velocities consensus of the vessels.

Substituting the control input (30) into (27), then

$\dot{V}=S^{T}$

$$
\begin{aligned}
& \times\left\{\alpha \operatorname{diag}\left(E_{2}^{\alpha-1}\right)\right. \\
& \times\left[\left((L+B) \otimes I_{3}\right) M^{-1}(\eta) \omega\right. \\
& -\left(B \otimes I_{3}\right)\left(1_{n} \otimes\left(M_{0}^{-1}\left(\eta_{0}\right) \tau_{0}\right)\right) \\
& -\operatorname{diag}\left\{\operatorname{diag}\left(M_{\min }^{-1}\left(2 n \omega_{\max }+b_{i} \bar{\tau}_{0}\right)+\kappa_{1}\right),\right. \\
& \left.\ldots, \operatorname{diag}\left(M_{\text {min }}^{-1}\left(2 n \omega_{\max }+b_{i} \bar{\tau}_{0}\right)+\kappa_{1}\right)\right\}
\end{aligned}
$$

$$
\begin{aligned}
& \times \operatorname{sign}(S)]\} \\
& =-\alpha \sum_{i=1}^{n}\left[s_{i}^{T}\left(e_{2}^{i}\right)^{\alpha-1}\right. \\
& \times \operatorname{diag}\left(M_{\min }^{-1}\left(2 n \omega_{\max }+b_{i} \bar{\tau}_{0}\right)+\kappa_{1}\right) \\
& \left.\times \operatorname{sign}\left(s_{i}\right)\right] \\
& +\alpha \sum_{i=1}^{n}\left[s_{i}^{T}\left(e_{2}^{i}\right)^{\alpha-1}\right. \\
& \times\left(\left(\sum_{j=1, j \neq i}^{n} a_{i j}+b_{i}\right)\left(M_{i}^{-1}\left(\eta_{i}\right)\left|\omega_{i}\right|\right)\right. \\
& \left.+\sum_{j=1, j \neq i}^{n} a_{i j}\left(M_{j}^{-1}\left(\eta_{j}\right)\left|\omega_{j}\right|\right)\right) \\
& \left.+b_{i} M_{i}^{-1}\left(\eta_{i}\right)\left|\tau_{0}\right|\right] \\
& \leq-\alpha \sum_{i=1}^{n}\left[\left|s_{i}^{T}\right|\left(e_{2}^{i}\right)^{\alpha-1}\right. \\
& \left.\times \operatorname{diag}\left(M_{\min }^{-1}\left(2 n \omega_{\max }+b_{i} \bar{\tau}_{0}\right)+\kappa_{1}\right) 1_{3}\right] \\
& +\alpha \sum_{i=1}^{n}\left[\left|s_{i}^{T}\right|\left(e_{2}^{i}\right)^{\alpha-1}\right. \\
& \times\left(\left(\sum_{j=1, j \neq i}^{n} a_{i j}+b_{i}\right)\left(M_{i}^{-1}\left(\eta_{i}\right)\left|\omega_{i}\right|\right)\right. \\
& \left.+\sum_{j=1, j \neq i}^{n} a_{i j}\left(M_{j}^{-1}\left(\eta_{j}\right)\left|\omega_{j}\right|\right)\right) \\
& \left.+b_{i} M_{i}^{-1}\left(\eta_{i}\right)\left|\tau_{0}\right|\right] \\
& \leq-\alpha \sum_{i=1}^{n}\left|s_{i}^{T}\right|\left(e_{2}^{i}\right)^{\alpha-1} \kappa_{1}
\end{aligned}
$$

Let

$$
\rho\left(E_{2}\right)=\min [\underbrace{\alpha\left(e_{2}^{1}(k)\right)^{\alpha-1} \kappa_{1}(k), \ldots \alpha\left(e_{2}^{n}(k)\right)^{\alpha-1} \kappa_{1}(k)}_{k=1,2,3}] .
$$




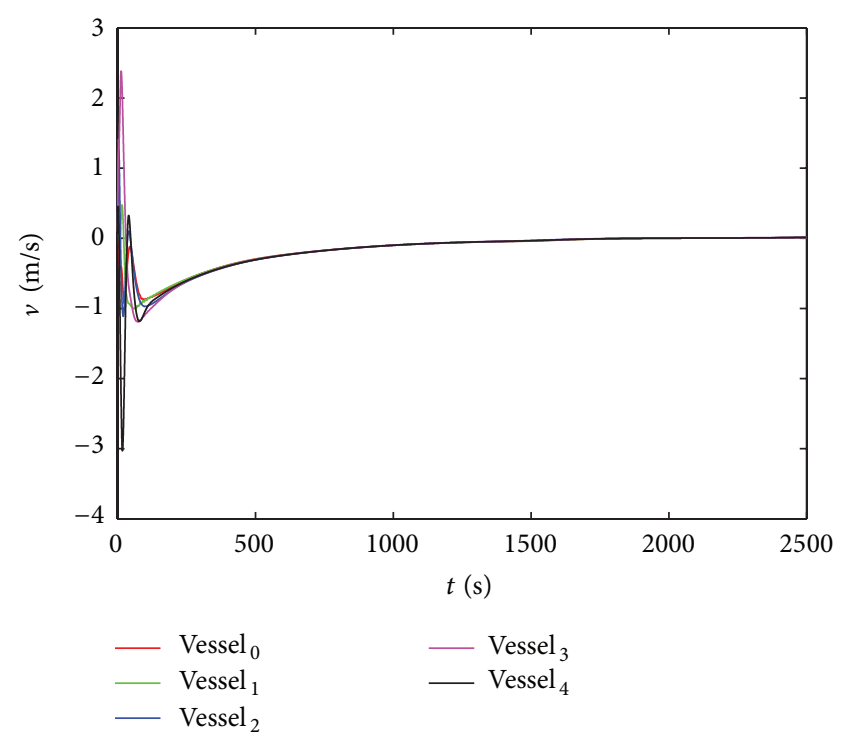

Figure 5: The sway velocities consensus of the vessels.

For $e_{2}^{k} \neq 0, k=1,2, \ldots, n, \rho\left(E_{2}\right)>0$, then we have

$$
\begin{aligned}
\dot{V} & \leq-\alpha \sum_{i=1}^{n}\left|s_{i}^{T}\right|\left(e_{2}^{i}\right)^{\alpha-1} \kappa_{1} \\
& \leq-\rho\left(E_{2}\right) \sum_{l=1}^{3 n}|S(l)| \\
& \leq-\rho\left(E_{2}\right)\left(\sum_{l=1}^{3 n}|S(l)|^{2}\right)^{1 / 2} \\
& =-2^{1 / 2} \rho\left(E_{2}\right) V^{1 / 2} .
\end{aligned}
$$

Therefore, we can know that the terminal sliding surface $S=0$ can be reached in a finite time for the case of $e_{2}^{k} \neq 0, k=$ $1,2, \ldots, n$, because the condition of finite-time stability is satisfied.

Substituting the control input (30) into the error dynamics (24), then

$$
\begin{aligned}
\dot{E}_{2}= & -\frac{\left(E_{2}\right)^{2-\alpha}}{\alpha} \\
& -\left(\operatorname{diag}\left(2 n \omega_{\max }+\kappa_{1}\right)+\left(B \otimes I_{3}\right)\left(1_{n} \otimes \tau_{0}\right)\right) \\
& \times \operatorname{sign}(S) \\
& +(L+B) \omega+\left(B \otimes I_{3}\right)\left(1_{n} \otimes \tau_{0}\right) .
\end{aligned}
$$

If $S \neq 0, \dot{e}_{2}^{i} \leq-\kappa_{1}$ or $\dot{e}_{2}^{i} \geq \kappa_{1}$, we can know that $E_{2}=0$ is not an attractor.

However, on this new terminal sliding-mode surface, that is, $S=0, E_{1}+E_{2}^{\alpha}=0$, so $E_{2}=-E_{1}^{1 / \alpha}$.

Define the Lyapunov function as

$$
V_{E_{1}}=\frac{1}{2} E_{1}^{T} E_{1}
$$

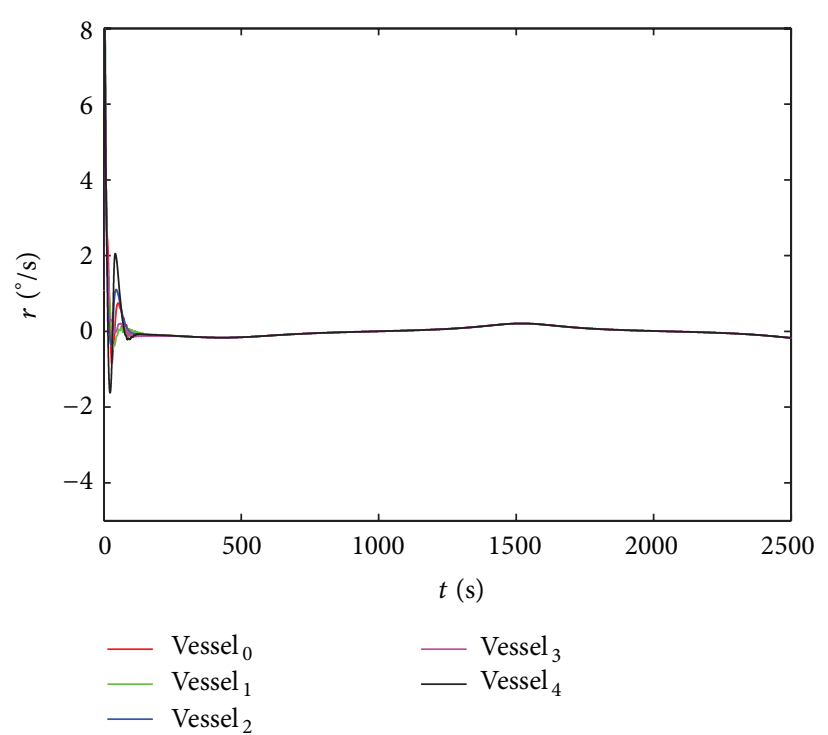

FIGURE 6: The angular velocities consensus of the vessels.

And it follows that

$$
\dot{V}_{E_{1}}=-E_{1}^{T} E_{1}^{1 / \alpha} \leq-2^{(1+\alpha) / 2 \alpha}\left(V_{E_{1}}\right)^{(1+\alpha) / 2 \alpha} .
$$

In light of Lemma 2, the error functions $E_{1}$ and $E_{2}$ will converge to zero in finite time.

\section{Simulation Results}

In this section, simulation results are presented to evaluate the performance of the proposed finite-time cooperative formation control algorithm. We consider four surface vessels to perform the cooperative tracking task. For detailed system parameters matrices of vessel mathematic model, we can refer to the literature [7]. Here, we suppose that the information of virtual leader is available only to vessel 3 and vessel 4 . The information exchange topology among all the vessels (including the virtual vessel) can be denoted as a directed graph in Figure 1.

From the above information exchange topology graph, we can know that the adjacent matrix of the graph is as follows:

$$
\bar{A}=\left[\begin{array}{lllll}
0 & 0 & 0 & 0 & 0 \\
0 & 0 & 1 & 1 & 1 \\
0 & 0 & 0 & 1 & 0 \\
1 & 0 & 1 & 0 & 0 \\
1 & 0 & 0 & 0 & 0
\end{array}\right] .
$$

Then the Laplacian matrix of the information exchange topology graph of the practical vessels can be written as

$$
L=\left[\begin{array}{cccc}
3 & -1 & -1 & -1 \\
0 & 1 & -1 & 0 \\
0 & -1 & 1 & 0 \\
0 & 0 & 0 & 0
\end{array}\right]
$$


And the matrix for the communication relationship between the virtual vessel and the practical vessels is

$$
B=\operatorname{diag}\left(\begin{array}{llll}
0 & 0 & 1 & 1
\end{array}\right) \text {. }
$$

The initial conditions are $\eta_{0}(0)=\left[\begin{array}{lll}27 & 782 & -\pi / 3\end{array}\right]^{T}$, $\eta_{1}(0)=\left[\begin{array}{lll}80 & 831 & -7 \pi / 30\end{array}\right]^{T}, \eta_{2}(0)=\left[\begin{array}{lll}-94 & 753 & -\pi / 2\end{array}\right]^{T}$, $\eta_{3}(0)=\left[\begin{array}{lll}40 & 700 & -\pi / 4\end{array}\right]^{T}$ and $\eta_{4}(0)=\left[\begin{array}{lll}-50 & 800 & -\pi / 3\end{array}\right]^{T}$, respectively. The desired trajectory for the virtual vessel is chosen as $\eta_{d}(t)=\left[\begin{array}{lll}n_{d} & e_{d} & \psi_{d}\end{array}\right]^{T}$, and the detailed expressions are $n_{d}=t, e_{d}=800 \sin (t / 800)$, and $\psi_{d}=\arctan \left(\dot{e}_{d} / \dot{n}_{d}\right)$. In order to maintain the desired formation pattern, the relative distance between the practical vessels and the virtual leader vessel is defined as $l_{1}=\left[\begin{array}{lll}0 & 100 & 0\end{array}\right]^{T}, l_{2}=\left[\begin{array}{lll}0 & -100 & 0\end{array}\right]^{T}$, $l_{3}=\left[\begin{array}{lll}0 & 50 & 0\end{array}\right]^{T}$, and $l_{4}=\left[\begin{array}{lll}0 & -50 & 0\end{array}\right]^{T}$, respectively.

With the proposed finite-time cooperative tracking control law, the dynamic trajectory of each vessel is shown in Figure 2. It can be seen that these vessels move collectively along the sinusoid with maintaining a beeline formation pattern in the plane. The heading consensus for these vessels is achieved in finite time as shown in Figure 3. Furthermore, in the process of cooperative tracking, the surge velocities, the sway velocities, and the angular velocities of all these surface vessels converge to the desired values as a whole in finite time, which are presented in Figures 4, 5, and 6, respectively. From Figure 4 , it is clearly seen that the surge velocities consensus of these vessels cannot be achieved absolutely at the inflexion of the curves. This is a natural phenomenon because all the desired trajectories are curve and all the curvatures are distinct.

Based on the above simulation results, we can know that the cooperative tracking task of multiple surface vessels is achieved by the proposed finite-time cooperative control algorithm. That means that these surface vessels can form the desired formation and perform the cooperative tracking as a whole formation in finite time. Overall, the proposed finitetime cooperative tracking control algorithm for multiple surface vessels is effective and satisfactory.

\section{Conclusion}

In this paper, the finite-time cooperative tracking control scheme for multiple surface vessels has been proposed. The cooperative formation is achieved by defining the formation reference point of each vessel based on the virtual leader-follower strategy. Furthermore, the communication topology among these vessels (include the virtual leader) is only the directed graph with a directed spanning tree. The cooperative tracking control scheme is designed using the terminal sliding-mode control approach which requires defining a nonlinear sliding variable function. In addition, the robustness against the external disturbances is achieved by compensating for the upper bound in the control input. It is proved that the cooperative tracking with desired formation can be achieved in finite time. Finally, the effectiveness of the proposed finite-time cooperative tracking control algorithm is validated by the simulation results.

\section{Conflict of Interests}

The authors declare that there is no conflict of interests regarding the publication of this paper.

\section{Acknowledgments}

The authors would like to acknowledge the support of the National Technology Momentous Special Program of China (2011ZX05027-002), High Technology of ships Research Program of China (Z12SJENA0011), the National Natural Science Fund of China (NSFC51209056), and the Basic Research Business Particular Item Fund of the Central High School of China (HEUCF041405).

\section{References}

[1] I. Ihle, Coordinated control of marine craft [Ph.D. thesis], Norwegian University of Science and Technology, 2006.

[2] W. Ren, R. W. Beard, and E. M. Atkins, "A survey of consensus problems in multi-agent coordination," in Proceedings of the American Control Conference (ACC '05), pp. 1859-1864, June 2005.

[3] F. Fahimi, "Non-linear model predictive formation control for groups of autonomous surface vessels," International Journal of Control, vol. 80, no. 8, pp. 1248-1259, 2007.

[4] I.-A. F. Ihle, J. Jouffroy, and T. I. Fossen, "Formation control of marine surface craft: a lagrangian approach," IEEE Journal of Oceanic Engineering, vol. 31, no. 4, pp. 922-934, 2006.

[5] F. Arrichiello, S. Chiaverini, and T. Fossen, "Formation control of underactuated surface vessels using the null-space-based behavioral control," in Proceedings of IEEE International Conference on Interlligent Robots and Systems, pp. 5942-5947, 2006.

[6] J. Almeida, C. Silvestre, and A. M. Pascoal, "Cooperative control of multiple surface vessels with discrete-time periodic communications," International Journal of Robust and Nonlinear Control, vol. 22, no. 4, pp. 398-419, 2012.

[7] C. Thorvaldsen and R. Skjetne, "Formation control of fullyactuated marine vessels using group agreement protocols," in Proceedings the 50th IEEE Conference on Decision and Control and European Control Conference, pp. 4132-4139, 2011.

[8] Y. Wang, W. Yan, and J. Li, "Passivity-based formation control of autonomous underwater vehicles," IET Control Theory \& Applications, vol. 6, no. 4, pp. 518-525, 2012.

[9] M. Fu and J. Jiao, "A hybrid approach for coordinated formation control of multiple surface vessels," Mathematical Problems in Engineering, vol. 2013, Article ID 794284, 8 pages, 2013.

[10] E. Kyrkjebø, K. Y. Pettersen, M. Wondergem, and H. Nijmeijer, "Output synchronization control of ship replenishment operations: theory and experiments," Control Engineering Practice, vol. 15, no. 6, pp. 741-755, 2007.

[11] M. Breivik, V. E. Hovstein, and T. I. Fossen, "Ship formation control: a guided leader-follower approach," in Proceedings of the 17th World Congress, International Federation of Automatic Control (IFAC '08), pp. 16008-16014, July 2008.

[12] N. E. Leonard and E. Fiorelli, "Virtual leaders, artificial potentials and coordinated control of groups," in Proceedings of the 40th IEEE Conference on Decision and Control (CDC '01), pp. 2968-2973, December 2001. 
[13] H. Shi, L. Wang, and T. Chu, "Virtual leader approach to coordinated control of multiple mobile agents with asymmetric interactions," Physica D, vol. 213, no. 1, pp. 51-65, 2006.

[14] W. Ren, "Consensus tracking under directed interaction topologies: algorithms and experiments," IEEE Transactions on Control Systems Technology, vol. 18, no. 1, pp. 230-237, 2010.

[15] W. Yu, G. Chen, and M. Cao, "Consensus in directed networks of agents with nonlinear dynamics," IEEE Transactions on Automatic Control, vol. 56, no. 6, pp. 1436-1441, 2011.

[16] W. Yu, G. Chen, M. Cao, and J. Kurths, "Second-Order consensus for multiagent systems with directed topologies and nonlinear dynamics," IEEE Transactions on Systems, Man, and Cybernetics B, vol. 40, no. 3, pp. 881-891, 2010.

[17] W. Zhang, Z. Wang, and Y. Guo, "Adaptive backstepping-based synchronization of uncertain networked Lagrangian systems," in Proceedings of the American Control Conference (ACC '11), pp. 1057-1062, July 2011.

[18] M. Fu, J. Jiao, J. Liu, and Y. Wang, "Coordinated formation control of nonlinear marine vessels under directed communication topology," in Proceedings of MTS/IEEE Conference on Oceans, pp. 1-7, 2013.

[19] S. P. Bhat and D. S. Bernstein, "Finite-time stability of continuous autonomous systems," SIAM Journal on Control and Optimization, vol. 38, no. 3, pp. 751-766, 2000.

[20] M. Fu, J. Jiao, and S. Yin, "Robust coordinated formation for multiple surface vessels based on backstepping sliding mode control," Abstract and Applied Analysis, vol. 2013, Article ID 681319, 10 pages, 2013.

[21] L. Wang and F. Xiao, "Finite-time consensus problems for networks of dynamic agents," IEEE Transactions on Automatic Control, vol. 55, no. 4, pp. 950-955, 2010.

[22] S. Khoo, L. Xie, and Z. Man, "Robust finite-time consensus tracking algorithm for multirobot systems," IEEE/ASME Transactions on Mechatronics, vol. 14, no. 2, pp. 219-228, 2009.

[23] F. Xiao, L. Wang, J. Chen, and Y. Gao, "Finite-time formation control for multi-agent systems," Automatica, vol. 45, no. 11, pp. 2605-2611, 2009.

[24] H. Du, S. Li, and X. Lin, "Finite-time formation control of multiagent systems via dynamic output feedback," International Journal of Robust and Nonlinear Control, vol. 23, no. 14, pp. 1609-1628, 2013.

[25] S. Li and X. Wang, "Finite-time consensus and collision avoidance control algorithms for multiple AUVs," Automatica, vol. 49, no. 11, pp. 3359-3367, 2013.

[26] S. Yin, S. Ding, and H. Luo, "Real-time implementation of fault tolerant control system with performance optimization," IEEE Transactions on Industrial Electronics, vol. 64, no. 5, pp. 24022411, 2014.

[27] S. Yin, G. Wang, and H. Karimi, "Data-driven design of robust fault detection system for wind turbines," Mechatronics, 2013.

[28] S. Yin, S. Ding, A. Haghani, H. Hao, and P. Zhang, "A comparison study of basic data-driven fault diagnosis and process monitoring methods on the benchmark Tennessee Eastman process," Journal of Process Control, vol. 22, no. 9, pp. 1567-1581, 2012.

[29] S. Yin, S. X. Ding, A. H. A. Sari, and H. Hao, "Data-driven monitoring for stochastic systems and its application on batch process," International Journal of Systems Science, vol. 44, no. 7, pp. 1366-1376, 2013.

[30] J. Huang, H. Li, Y. Chen, and Q. Xu, "Robust position control of PMSM using fractional-order sliding mode controller," Abstract and Applied Analysis, vol. 2012, Article ID 512703, 33 pages, 2012.
[31] E. A. Tannuri, A. C. Agostinho, H. M. Morishita, and L. Moratelli, "Dynamic positioning systems: an experimental analysis of sliding mode control," Control Engineering Practice, vol. 18, no. 10, pp. 1121-1132, 2010.

[32] H. Ashrafiuon, K. R. Muske, L. C. McNinch, and R. A. Soltan, "Sliding-mode tracking control of surface vessels," IEEE Transactions on Industrial Electronics, vol. 55, no. 11, pp. 40044012, 2008.

[33] F. Fahimi, "Sliding-mode formation control for underactuated surface vessels," IEEE Transactions on Robotics, vol. 23, no. 3, pp. 617-622, 2007.

[34] M. Defoort, T. Floquet, A. Kökösy, and W. Perruquetti, "Slidingmode formation control for cooperative autonomous mobile robots," IEEE Transactions on Industrial Electronics, vol. 55, no. 11, pp. 3944-3953, 2008.

[35] D. Zhao and T. Zou, "A finite-time approach to formation control of multiple mobile robots with terminal sliding mode," International Journal of Systems Science, vol. 43, no. 11, pp. 19982014, 2012.

[36] T. Fossen, Marine Control Systems: Guidance, Navigation and Control of Ships, Rigs and Underwater Vehicles, Marine Cybernetics, Trondheim, Norway, 2002.

[37] S. P. Bhat and D. S. Bernstein, "Finite-time stability of continuous autonomous systems," SIAM Journal on Control and Optimization, vol. 38, no. 3, pp. 751-766, 2000. 


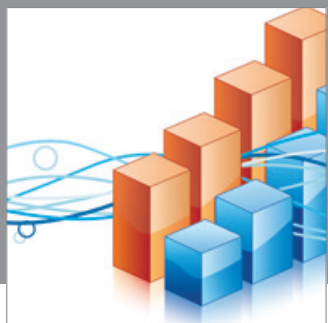

Advances in

Operations Research

mansans

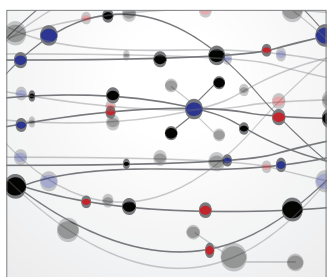

The Scientific World Journal
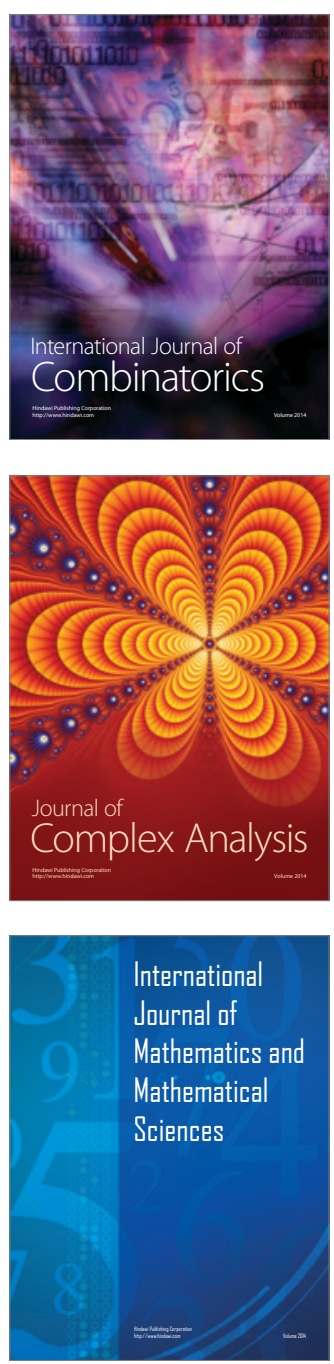
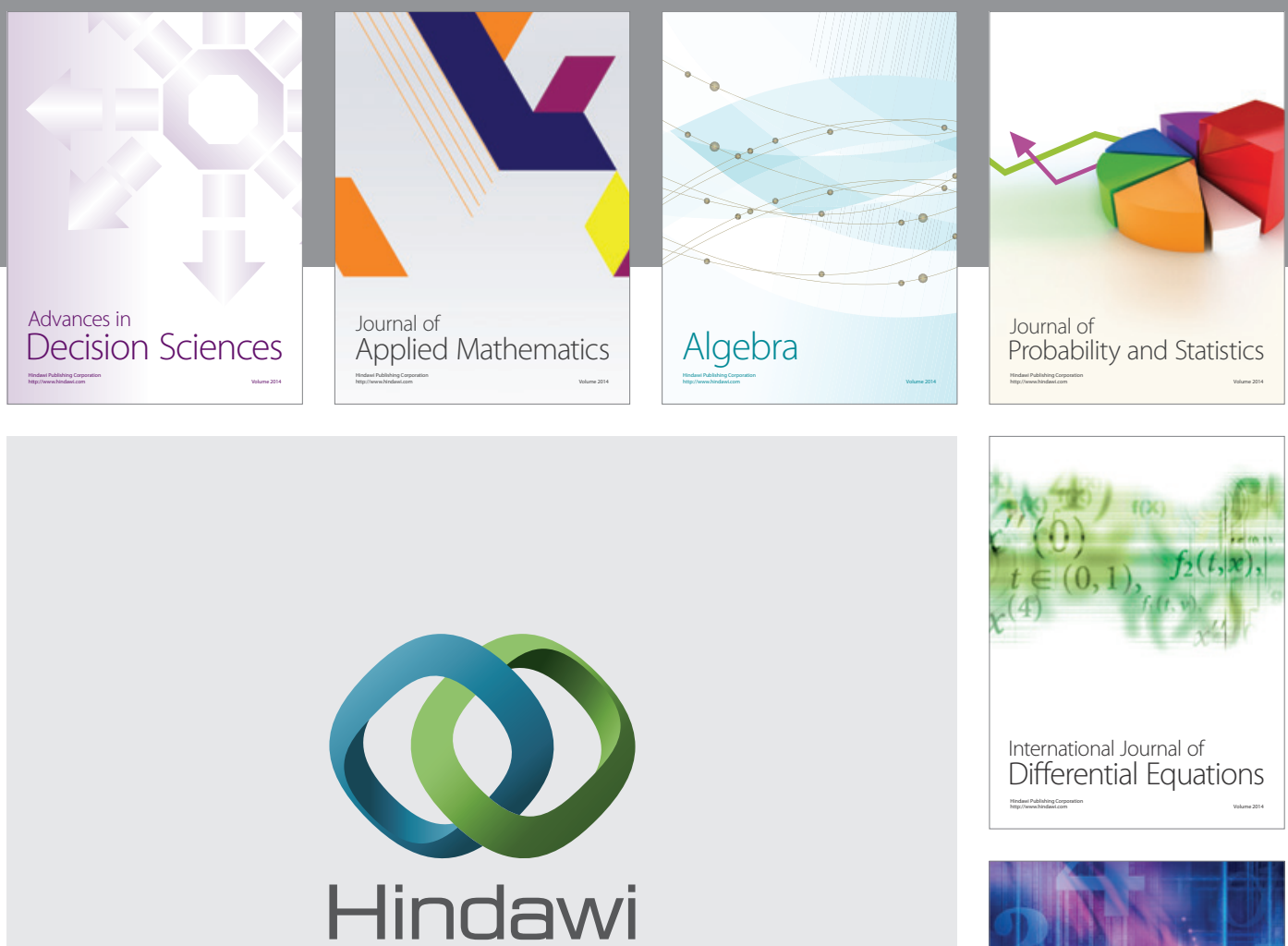

Submit your manuscripts at http://www.hindawi.com
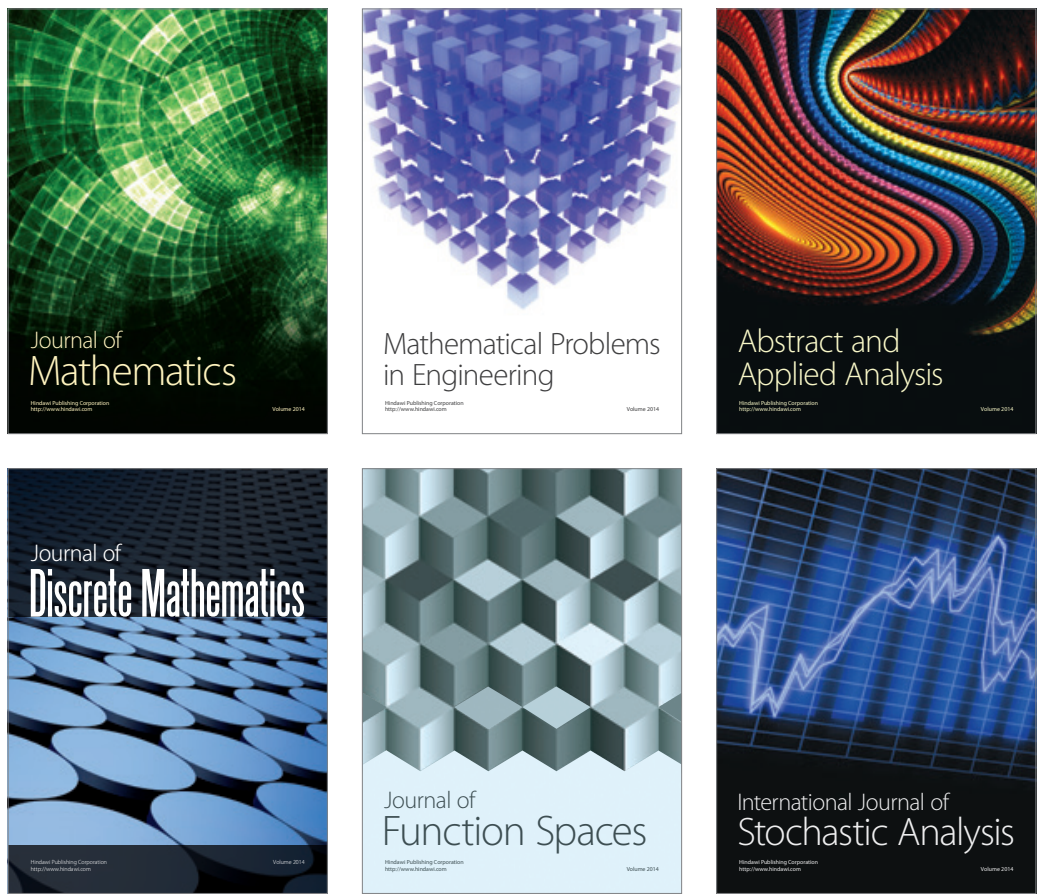

Journal of

Function Spaces

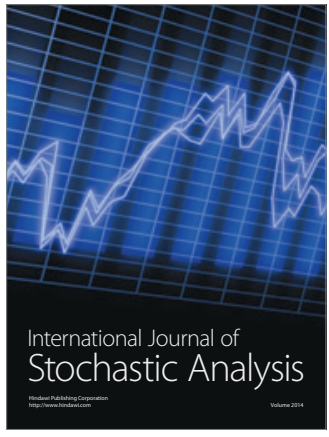

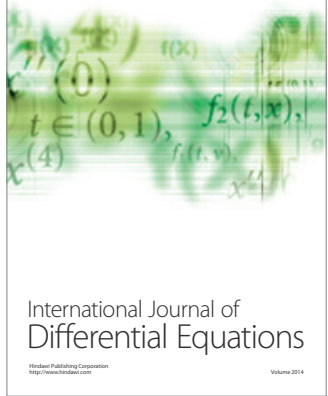
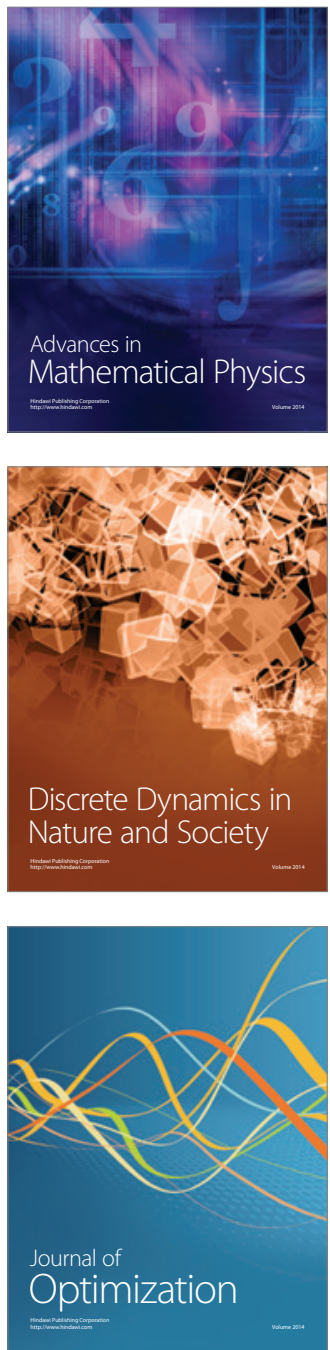